\begin{abstract}
MODERATE-severe depression (MSD) is linked to overexpression of proinflammatory cytokines and chemokines. Fractalkine (FKN) and macrophage inflammatory protein-1 alpha (MIP-1 $\alpha$ ) are, respectively, members of $\mathrm{CX} 3 \mathrm{C}$ and $\mathrm{C}-\mathrm{C}$ chemokines, and both are involved in recruiting and activating mononuclear phagocytes in the central nervous system. We analysed the presence of FKN and MIP-1 $\alpha$ in sera of untreated MSD patients and healthy donors. High FKN levels were observed in all MSD patients as compared with values only detectable in $26 \%$ of healthy donors. MIP-1 $\alpha$ was measurable in $20 \%$ of patients, while no healthy donors showed detectable chemokine levels. In conclusion, we describe a previously unknown involvement of FKN in the pathogenesis of MSD, suggesting that FKN may represent a target for a specific immune therapy of this disease.
\end{abstract}

Key words: Moderate-severe depression, fractalkine, Macrophage inflammatory protein-1 alpha, Serum

\section{Involvement of fractalkine and macrophage inflammatory protein-1 alpha in moderate- severe depression}

\author{
Rosaria Alba Merendino ${ }^{1}$, Giuseppe Di Pasquale ${ }^{2}$, \\ Filippo De Luca², Laura Di Pasquale², \\ Edoardo Ferlazzo ${ }^{3}$, Gabriella Martino4, \\ Maura Carmela Palumbo ${ }^{5}$, Fortunato Morabito ${ }^{6}$ and \\ Sebastiano Gangemi ${ }^{7, C A}$
}

${ }^{1}$ Chair of Immunopathology, Department of Human Pathology, ${ }^{2}$ Department of Pediatrics Science,

${ }^{3}$ School and Division of Neurology, Department of Neurosciences, and ${ }^{4}$ Department of Neurosciences, Psychiatry and Anesthesiology, University of Messina, Italy; ${ }^{5}$ SPDC, and ${ }^{6}$ Bone Marrow Transplant Unit, Azienda Ospedaliera Bianchi-Melacrino-Morelli, Reggio Calabria, Italy; ${ }^{7}$ School of Allergy and Clinical Immunology, Department of Human Pathology, University of Messina

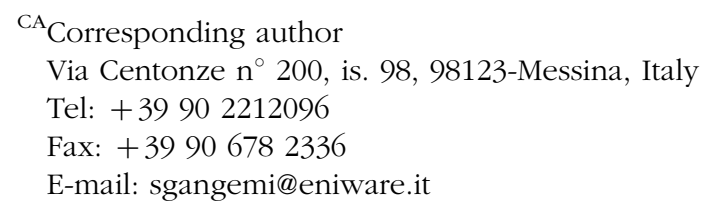

Depression represents a major public health problem. The interactions between the central nervous system (CNS) and the immune system in depression have a biological explanation supported by the monoamine hypothesis, deregulation of hypothalamic-pituitary-adrenocortical activity, and the macrophage theory, which proposes that an excessive secretion of macrophage cytokines such as interleukin-1 may provoke the hormonal abnormalities linked to depression. ${ }^{1}$

In the superfamily of cytokines, the chemokines appear as a rapidly evolving family of intercellular mediators, with their number and variety growing. These key factors in the immune response have been associated with the trafficking of leukocytes in physiological immune surveillance as well as with inflammatory cell recruitment in different diseases. $^{2}$

In the CNS, chemokine signalling plays an important role in neuropathological processes, in addition to the relevant role played under physiological processes, such as neuronal migration and modulation of synaptic transmission, conditions. ${ }^{2}$

The $\mathrm{CX} 3 \mathrm{C}$ or $\delta$-chemokines include only one member, called CX3CL1/fractalkine (FKN), in which the first two cysteines are separated by three amino acids. This molecule exists in both membraneanchored and soluble isoforms. ${ }^{2}$

FKN is highly expressed in the CNS, being localized on neuronal cells, while its receptor, CX3CR1, is exclusively found on microglia and astrocytes. ${ }^{2}$

The finding of a constitutive and abundant neuronal mRNA expression for FKN favours the view of more physiological functions of FKN such as the mediation of adhesion and cell-cell communication between FKN-producing neurones and microglia that express FKN receptor. This cellular cross-talk may also occur in the total absence of pro-inflammatory conditions. $^{2}$

The CC or $\beta$-chemokines contain four conserved cysteines, the first two of which are adjacent. Macrophage inflammatory protein-1 alpha (MIP-1 $\alpha)$, 
a member of $\mathrm{C}-\mathrm{C}$ or $\beta$-chemokines, has been implicated in the pathogenesis of several human diseases with inflammatory components. ${ }^{2}$ MIP- $1 \alpha$ plays a key role in recruiting and activating mononuclear phagocytes in the CNS, microglia and astrocytes being sources of this chemokine. ${ }^{2}$

Suarez et al. have reported that men with mild to moderate levels of depressive symptoms showed overexpression of monocyte-associated proinflammatory cytokines and chemokines. ${ }^{3}$

In line with these findings, since no data on a possible association of FKN and MIP- $1 \alpha$ release and depression disease are currently available, we investigated FKN and MIP- $1 \alpha$ serum levels in patients affected by moderate-severe depression (MSD), before any anti-depression therapy.

In this study, we enrolled nine female patients with MSD (mean age, $36.4 \pm 12.1$ years) who experienced a major depressive episode according to DSM-IV criteria, recruited from the Outpatient Mental Health Service of the Department of Neurosciences, Psychiatry and Anesthesiology. Mood was assessed at baseline using the Beck Depression Inventory (range, 0-63). The mean baseline score of the Beck Depression Inventory was 30.2. Fifteen healthy females (mean age, 34.7 \pm 11.3 years) were also enrolled as controls. Patients and healthy donors (HD) signed an informed consent form.

Sera were obtained from peripheral blood allowed to clot at room temperature for $2 \mathrm{~h}$, separated by centrifugation at $200 \times \mathrm{g}$ for $15 \mathrm{~min}$ in a $4235 \mathrm{~A}$ centrifuge (ALC Int. S.r.L., Milan, Italy), and stored at $-80^{\circ} \mathrm{C}$ until use.

Soluble FKN was measured in an enzyme-linked immunosorbent assay, using DUOSET from R\&D System Inc. (SPACE Import-Export, Milan, Italy). The sensitivity of $156 \mathrm{pg} / \mathrm{ml}$ was obtained by gentle plate agitation during all incubation steps.

Serum MIP-1 $\alpha$ levels were determined by an immunoenzymatic method (Quantikine Human MIP- $1 \alpha$; R\&D System Inc., SPACE Import-Export). The minimum detectable dose of MIP- $1 \alpha$ is typically less than $10 \mathrm{pg} / \mathrm{ml}$.

Data are expressed as the mean \pm standard deviation. $p<0.05$ was considered significant.

We found high levels of FKN in all nine MSD patients $(1078.2 \pm 302.3 \mathrm{pg} / \mathrm{ml}$ ) (Table 1 ) and in only $4 / 15(26 \%) \mathrm{HD}$ at lower concentrations $(212.5 \pm 63.5$ $\mathrm{pg} / \mathrm{ml}$ ). Detectable levels of MIP- $1 \alpha$ were found in 2/9 (22\%) MSD patients (56 and $72 \mathrm{pg} / \mathrm{ml}$, respectively) (Table 1) and no detectable levels in HD sera.

There is clinical and experimental evidence of severe alterations of the immune and endocrine systems linked to depression. In particular, it has been shown that the activation of the brain chemokine system is associated with depression, although
Table 1. Serum levels of FKN and MIP- $1 \alpha$ in patients affected by MSD

\begin{tabular}{lcl}
\hline Patient number & FKN $(\mathrm{pg} / \mathrm{ml})$ & MIP- $1 \alpha(\mathrm{pg} / \mathrm{ml})$ \\
\hline 1 & 813 & Not detected \\
2 & 1262 & Not detected \\
3 & 554 & 72 \\
4 & 1124 & 56 \\
5 & 1179 & Not detected \\
6 & 1201 & Not detected \\
7 & 781 & Not detected \\
8 & 1529 & Not detected \\
9 & 1261 & Not detected \\
\hline
\end{tabular}

the exact relationship between sickness behaviour and depression is still elusive. ${ }^{3}$

The results of this preliminary study, which is the first investigating the relationship between FKN and MIP- $1 \alpha$ with MSD, show high FKN serum levels in all patients and detectable values of FKN in 4/15 (26\%) HD only, and detectable levels of MIP- $1 \alpha$ in only $20 \%$ patients and no detectable levels in HD sera.

Thus, we can hypothesize an involvement of FKN release in the pathogenesis of MSD disease.

As previously reported by other investigators, our data support the demonstration that MSD disease is characterized by an increased immune response activity and that the systemic immune stimulation participates in the pathogenesis of this disease.

In a previous study, we demonstrated that interleukin-18, which promotes $\mathrm{T}$ helper (Th) 1 responses, is elevated in patients affected by MSD, whereas sCD30, expressed by activated Th2, is undetectable in the same patients. ${ }^{4}$ In this respect, it has been shown that chemokines are an important component of polarized type I and type II responses. ${ }^{5}$

Fraticelli et al. demonstrated that CX3CR1 is strongly expressed in natural killer cells and, at lower levels, in polarized Th1 cells, which respond chemotactically to FKN, unlike Th2 populations, contrary to other chemokines such as MIP-1 $\alpha$ (CCR1 agonist) that possess a comparable activity for Th1 and Th2 cells. These data suggest that FKN and its cognate receptor CX3CR1 are part of the chemokine-based amplification and orientation circuits of polarized Th1 responses. ${ }^{5}$

Therefore, our study strengthens the hypothesis that MDS disease is associated with an activation of cellular immunity and an induction of inflammatory response, mainly Th1 type mediated, at least in part, by FKN. Further studies are needed to clarify the relationship between the pathophysiology of MDS and elevated FKN levels.

In conclusion, our preliminary findings provide new insight into the immunopathogenesis of MDS, and suggest that FKN may represent a target for a specific immune therapy for this disease. 
ACKNOWLEDGEMENTS. This work was supported in part by a grant from University of Messina, Medical School, Italy.

\section{References}

1. Nunes SOV, Reiche EMV, Morimoto HK, et al. Immune and hormonal activity in adults suffering from depression. Braz J Med Biol Res 2002; 35 $581-587$.

2. Bajetto A, Bonavia R, Barbero S, Schettini G. Characterization of chemokines and their receptors in the central nervous system: physiopathological implications. J Neurochem 2002; 82: 1311-1329.
3. Suarez EC, Krishnan RR, Lewis JG. The relation of severity of depressive symptoms to monocyte-associated proinflammatory cytokines and chemokines in apparently healthy men. Psychosom Med 2003; 65: 362-368.

4. Merendino RA, Di Rosa AE, Di Pasquale G, et al. Interleukin-18 and CD30 serum levels in patients with moderate-severe depression. Mediat Inflamm 2002; 11: 265-267.

5. Fraticelli P, Sironi M, Bianchi G, et al. Fractalkine (CX3CL1) as an amplification circuit of polarized Th1 responses. J Clin Invest 2001; 107: $1173-1181$

\section{Received 6 February 2004}

Accepted 12 March 2004 


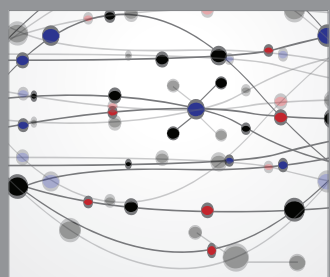

The Scientific World Journal
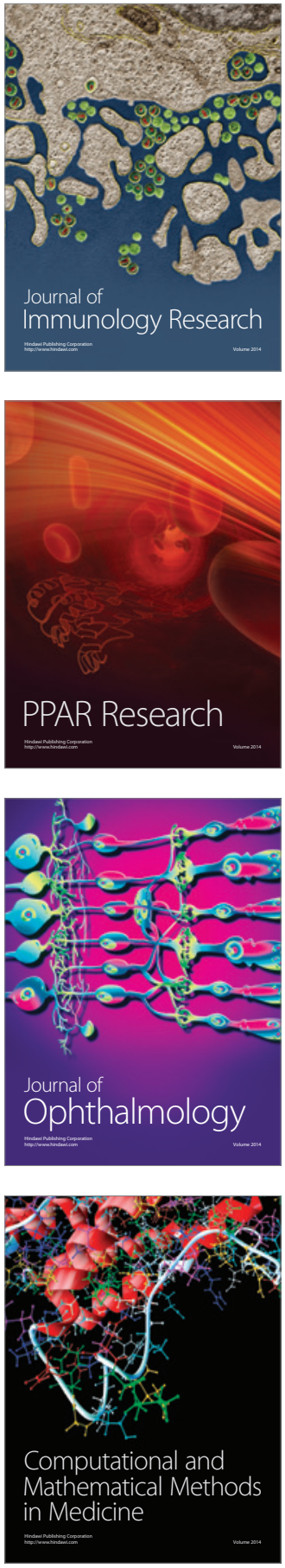

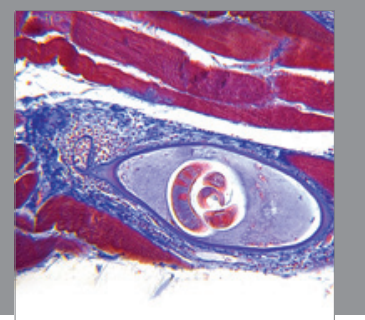

Gastroenterology

Research and Practice
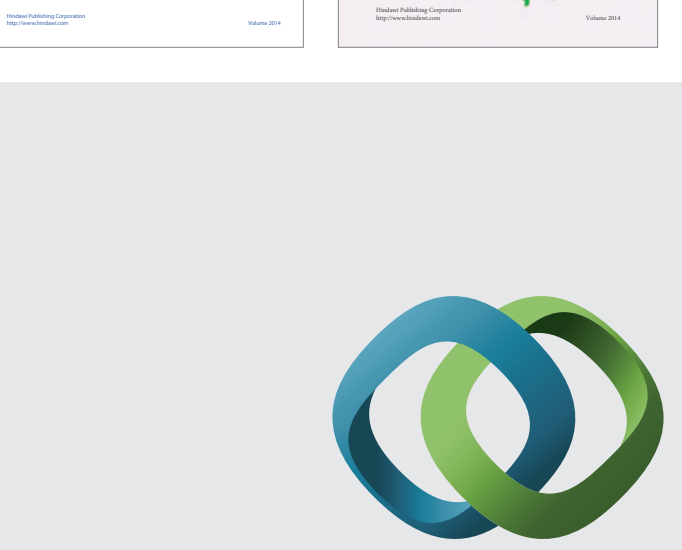

\section{Hindawi}

Submit your manuscripts at

http://www.hindawi.com
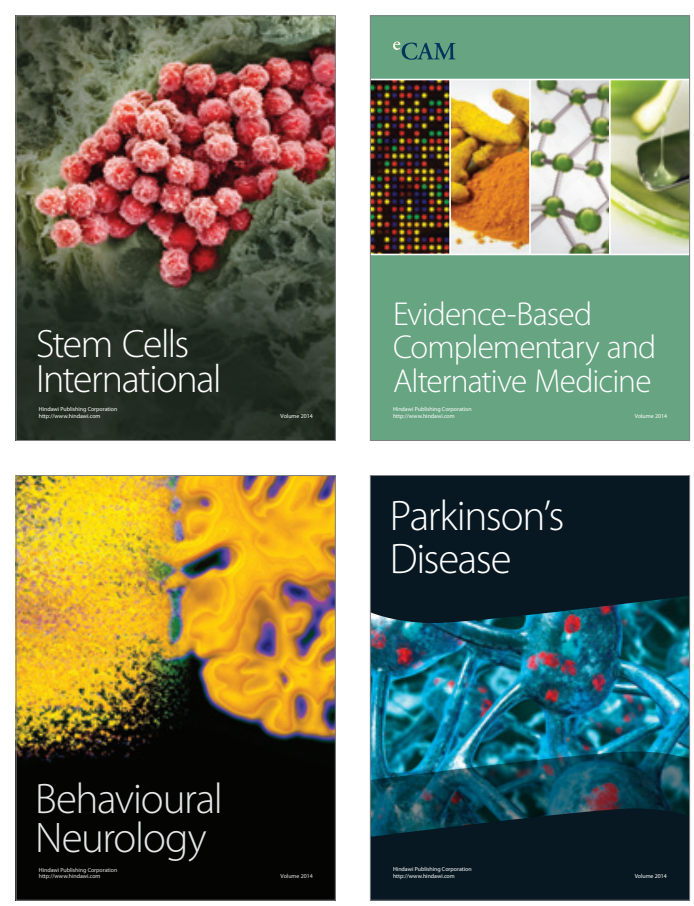

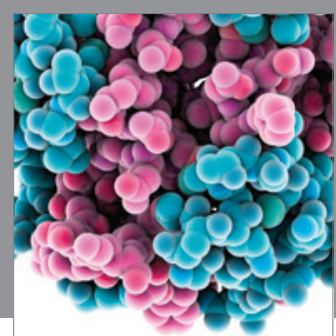

Journal of
Diabetes Research

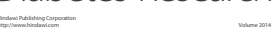

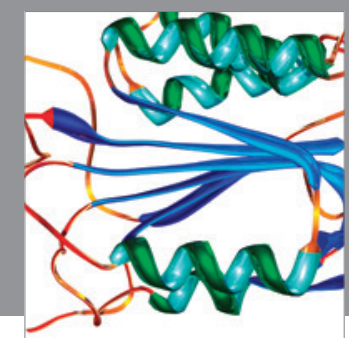

Disease Markers
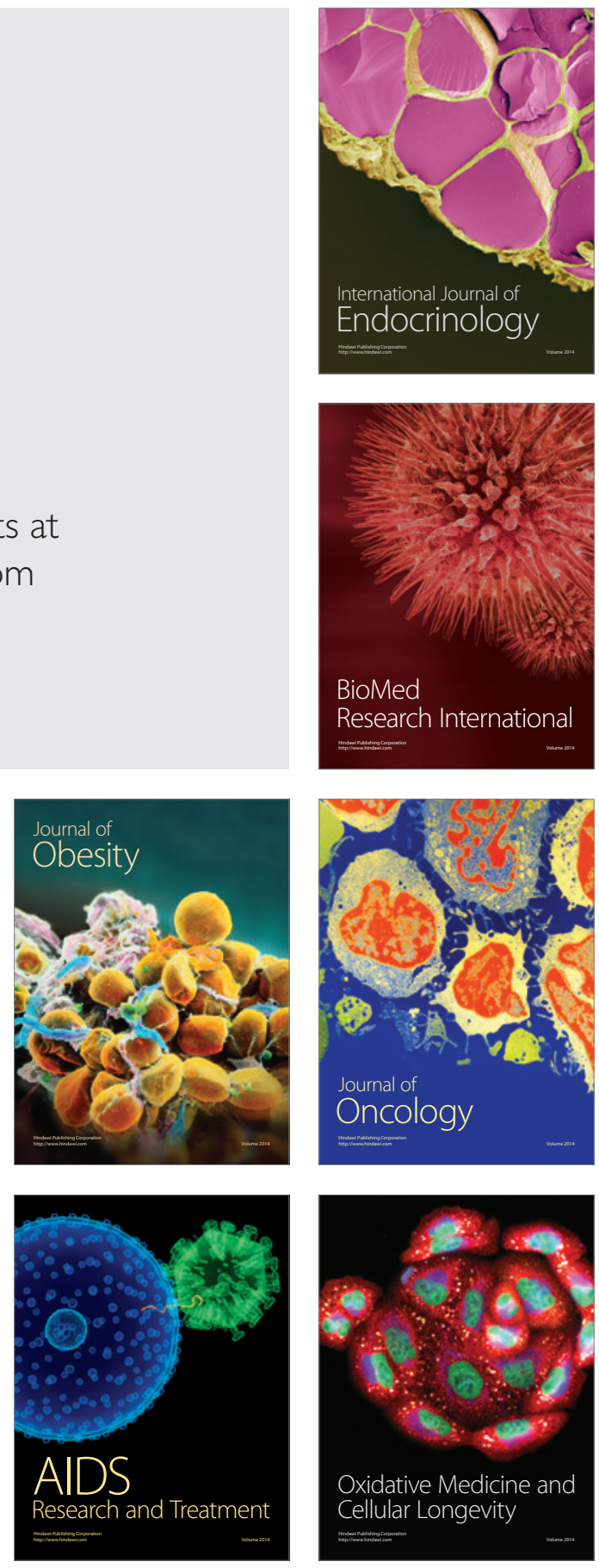